doi: $10.13108 / 2021-13-2-160$

\title{
ON DARBOUX NON-INTEGRABILITY OF HIETARINTA EQUATION
}

\author{
S.YA. STARTSEV
}

\begin{abstract}
The autonomous Hietarinta equation is a well-known example of the quadgraph discrete equation which is consistent around the cube. In a recent work, it was conjectured that this equation is Darboux integrable, that is, for each of two independent discrete variables there exist non-trivial functions that remain unchanged on solutions of the equation after the shift in this discrete variable. We demonstrate that this conjecture is not true for generic values of the equation coefficients.

To do this, we employ two-point invertible transformations introduced by R.I. Yamilov. We prove that an autonomous difference equation on the quad-graph cannot be Darboux integrable if a transformation of the above type maps solutions of this equation into its solutions. This implies that the generic Hietarinta equation is not Darboux integrable since the Hietarinta equation in the general case possesses the two-point invertible autotransformations. Along the way, all Darboux integrable subcases of the Hietarinta equation are found. All of them are reduced by point transformations to already known integrable equations.

At the end of the article, we also briefly describe another way to prove the Darboux non-integrability of the Hietarinta equation. This alternative way is based on the known fact that a difference substitution relates this equation to a linear one. Thus, the Hietarinta equation gives us an example of a quad-graph equation that is linearizable but not Darboux integrable.
\end{abstract}

Keywords: Hietarinta equation, quad-graph equation, Bäcklund auto-transformation, Darboux integrability, C-integrability.

Mathematics Subject Classification: 39A14, 37K05, 37K10, 37K35

\section{INTRODUCTION}

The Hietarinta equation

$$
\frac{z_{n, m}+b}{z_{n, m}+a} \frac{z_{n+1, m+1}+d}{z_{n+1, m+1}+c}=\frac{z_{n+1, m}+b}{z_{n+1, m}+c} \frac{z_{n, m+1}+d}{z_{n, m+1}+a}, \quad n, m \in \mathbb{Z},
$$

where the constants $a, b, c$ and $d$ satisfy the inequality $(a-b)(a-d)(c-b)(c-d) \neq 0$, gives an important example of the quad-graph difference equation which is consistent around the cube [1]. This example was introduced in [2] to illustrate that the tetrahedron property is not necessary for the consistency around the cube.

A non-autonomous version of the Hietarinta equation was recently studied in [3]. It turned out that this non-autonomous version was Darboux integrable. At the very end of [3], the authors raised the question of whether autonomous Hietarinta equation (1.1) is Darboux integrable as well. The main purpose of the present article is to answer this question and to demonstrate that this answer is negative for the generic values of the constants $a, b, c$ and $d$.

S.Ya. Startsev, On Darboux non-integrability of the Hietarinta equation.

(C) S.Ya. Startsev. 2021.

Submitted February 21, 2021. 
Let us recall that an equation of the form

$$
u_{n+1, m+1}=F\left(u_{n, m}, u_{n+1, m}, u_{n, m+1}\right), \quad \frac{\partial F}{\partial u_{n+1, m}} \frac{\partial F}{\partial u_{n, m+1}} \frac{\partial F}{\partial u_{n, m}} \neq 0
$$

is called Darboux integrable if there exist functions $\Omega_{n, m}\left(u_{n, m}, u_{n+1, m}, \ldots, u_{n+p, m}\right), p>0$, and $\bar{\Omega}_{n, m}\left(u_{n, m}, u_{n, m+1}, \ldots, u_{n, m+\bar{p}}\right), \bar{p}>0$, such that they essentially depend on $u_{n, m}$ and their last arguments $\left(u_{n+p, m}\right.$ for $\Omega_{n, m}$ and $u_{n, m+\bar{p}}$ for $\left.\bar{\Omega}_{n, m}\right)$ and satisfy the relations

$$
\begin{aligned}
& \Omega_{n, m+1}\left(u_{n, m+1}, u_{n+1, m+1}, \ldots, u_{n+p, m+1}\right)=\Omega_{n, m}\left(u_{n, m}, u_{n+1, m}, \ldots, u_{n+p, m}\right), \\
& \bar{\Omega}_{n+1, m}\left(u_{n+1, m}, u_{n+1, m+1}, \ldots, u_{n+1, m+\bar{p}}\right)=\bar{\Omega}_{n, m}\left(u_{n, m}, u_{n, m+1}, \ldots, u_{n, m+\bar{p}}\right)
\end{aligned}
$$

for all $n, m$ and each solution $u$ of the equation. In other words, $\Omega_{n, m}$ and $\bar{\Omega}_{n, m}$ remain unchanged on solutions of $(1.2)$ after the shifts in $m$ and $n$, respectively. The functions $\Omega_{n, m}$ and $\bar{\Omega}_{n, m}$ are respectively called an $n$-integral of order $p$ and an $m$-integral of order $\bar{p}$ for the equation $(1.2)$.

It should be stressed that we eliminate all variables of the form $u_{n+i, m+j}, i \cdot j \neq 0$, via equation 1.2 to check whether a relation (in particular, relations (1.3), (1.4) ) holds on solutions of the equation. That is, we substitute $F\left(u_{n, m}, u_{n+1, m}, u_{n, m+1}\right)$ for $u_{n+1, m+1}$,

$$
F\left(u_{n+1, m}, u_{n+2, m}, F\left(u_{n, m}, u_{n+1, m}, u_{n, m+1}\right)\right) \text { for } u_{n+2, m+1}
$$

and so on. A relation holds on solutions of the equation if and only if this relation holds identically after the above substitutions. These substitutions show why we can assume without loss of generality that the integrals are independent of the variables of the form $u_{n+i, m+j}, i \cdot j \neq 0$. In addition, after eliminating these variables from the defining relations of the integrals, the independence of $\Omega_{n, m}$ and $\bar{\Omega}_{n, m}$ of the variables $u_{n, m+j}$ and $u_{n+i, m}$ follows directly from (1.3) and (1.4), respectively; see, for example, Lemma 1 in [4] for more details.

Remark 1.1. We note that defining relation (1.3) can be considered for each $n$ separately, and the corresponding $\Omega_{n, m}$ may be chosen quite differently for different $n$. For example, we can set $\Omega_{n, m}$ equal to constants for some values of $n$ and to non-constant 'solutions' of (1.3) (if they exist) for other $n$. But, since (1.2) is explicitly independent of $n$, we can choose $\Omega_{n, m}$ same for all $n$ and assume without loss of generality that it is explicitly independent of $n$, too. Under this assumption and by using the inequality in $(1.2)$, it can be proved that $\Omega_{n, m}$ must depend on its first and last arguments for all $n$ and $m$ if $\Omega_{n, m}$ depends on these arguments for at least some $m$ and satisfies (1.3) for all $m$. Of course, the same (up to the interchange $n \leftrightarrow m$ ) is also true for (1.4) and $\bar{\Omega}_{n, m}$.

A simple example of Darboux integrable equation is the 'multiplicative' discrete wave equation

admitting the integrals

$$
u_{n+1, m+1}=\frac{u_{n+1, m} u_{n, m+1}}{u_{n, m}}
$$

$$
\Omega_{n, m}=\frac{u_{n+1, m}}{u_{n, m}}, \quad \bar{\Omega}_{n, m}=\frac{u_{n, m+1}}{u_{n, m}} .
$$

We can modify (1.5) by the point transformation $\tilde{u}_{n, m}=\zeta_{n, m} u_{n, m}$, where $\zeta_{n, m}=-1$ if both $n$ and $m$ are odd, and $\zeta_{n, m}=1$ otherwise. This gives the autonomous equation

$$
\tilde{u}_{n+1, m+1}=-\frac{\tilde{u}_{n+1, m} \tilde{u}_{n, m+1}}{\tilde{u}_{n, m}}
$$

that admits the integrals

$$
\Omega_{n, m}=(-1)^{m} \frac{\tilde{u}_{n+1, m}}{\tilde{u}_{n, m}}, \quad \bar{\Omega}_{n, m}=(-1)^{n} \frac{\tilde{u}_{n, m+1}}{\tilde{u}_{n, m}} .
$$


We observe that these integrals are non-autonomous, i.e., they explicitly depend on $m$ and $n$. The last example looks contrived since the equations (1.5) and (1.6) are, in fact, same. However, some other autonomous quad-graph equations with non-autonomous integrals can be found, for example, in [5]. Thus, we may not exclude non-autonomous integrals from consideration when we check an autonomous equation for the Darboux integrability.

It is interesting that the non-autonomous Hietarinta equation in [3] is reduced to (1.6) by a non-autonomous Möbius transformations, while the work [2] relates the equation (1.1) to (1.5) by a point transformation in the case $|a-c|+|b-d|=0$ only. A less trivial example of Darboux integrable discrete equation is the equation

$$
\frac{u_{n+1, m+1}}{u_{n, m+1}}=\frac{u_{n+1, m}+1}{u_{n, m}+1}
$$

admitting the integrals

$$
\frac{u_{n, m+1}}{u_{n, m}+1} \quad \text { and } \quad \frac{u_{n+2, m}-u_{n+1, m}}{u_{n+1, m}-u_{n, m}} .
$$

This equation and its integrals were found in [5]. We show below that a point transformation reduce equation (1.1) to (1.7) in the case $a=c, b \neq d$ and to the equation obtained from (1.7) by interchanging $n \leftrightarrow m$ in the case $a \neq c, b=d$. Hence, equation (1.1) is Darboux integrable if $(a-c)(b-d)=0$. In the present paper, we prove that all other cases of the autonomous Hietarinta equation (1.1) are not Darboux integrable and, moreover, do not admit an integral even in one direction.

As it was demonstrated in [6], 17], the smallest orders of the integrals for Darboux integrable equations can be arbitrary high. To prove Darboux non-integrability, we therefore need to make sure that there are no integrals of order $p$ for all $p$. Characteristic algebras [8] and Laplace invariants [9], [10] give strong and enough constructive necessary conditions for the existence of integrals of order $p$ for the quad-graph equations, but these methods do not solve the problem of the arbitrariness of $p$. This is why the Darboux non-integrability of a quad-graph equation is not always obvious.

To demonstrate the absence of integrals for the generic equation (1.1), we employ some specific properties of the Heitarinta equation and prove theorems about the absence of integrals for any quad-graph equation with the same properties 1 . These properties are considered in the next section.

\section{Hietarinta equation: transformations AND DARBOUX INTEGRABle SUbCASES}

Following the work [11], it is convenient to make the point transformation

$$
z_{n, m}=\frac{d-c u_{n, m}}{u_{n, m}-1}
$$

in the equation (1.1), that is, we denote $\frac{z_{n, m}+d}{z_{n, m}+c}$ by $u_{n, m}$ and rewrite (1.1) in terms of this new variable. In the case $a \neq c$ this gives the equation

$$
u_{n+1, m+1}\left(u_{n, m}+B\right)\left(u_{n, m+1}+A\right)=u_{n, m+1}\left(u_{n+1, m}+B\right)\left(u_{n, m}+A\right),
$$

where $A=(d-a) /(a-c)$ and $B=(d-b) /(b-c)$. It should be noted that $A \neq B$ because

$$
A-B=\frac{(d-c)(b-a)}{(a-c)(b-c)}
$$

and the coefficients of (1.1) satisfy the conditions $d \neq c$ and $b \neq a$.

\footnotetext{
${ }^{1}$ But the author does not know other nonlinear examples of quad-graph equations with these properties.
} 
If $a=c$, then (2.1) maps Hietarinta equation (1.1) into the equation

$$
\frac{u_{n+1, m+1}}{u_{n, m+1}}=\frac{u_{n+1, m}+B}{u_{n, m}+B},
$$

where the constant $B$ again equals $(d-b) /(b-c)$. The last equation coincides with (1.5) if $B=0$. Otherwise, the scale transformation $u_{n, m} \rightarrow B u_{n, m}$ relates this equation to (1.7).

In the case $B=0$, the equation $(2.2)$ becomes

$$
u_{n+1, m+1} \frac{u_{n, m+1}+A}{u_{n, m+1}}=u_{n+1, m} \frac{u_{n, m}+A}{u_{n, m}} .
$$

Recall that $d \neq a$ for (1.1) and $A \neq 0$ for 2.2 . Denoting $A / u_{n, m}$ by $v_{n, m}$, we obtain

$$
\frac{v_{n+1, m+1}}{v_{n+1, m}}=\frac{v_{n, m+1}+1}{v_{n, m}+1} \text {. }
$$

The last equation is Darboux integrable because it coincides with (1.7) up to the change of notation $v \rightarrow u$ and the interchange $n \leftrightarrow m$.

Summarizing the results of three previous paragraphs, we arrive at the following statement.

Proposition 2.1. Hietarinta equation (1.1) is Darboux integrable if $(a-c)(b-d)=0$. In all other cases, point transformation (2.1) reduces (1.1) to equation (2.2) with non-zero constants $A$ and $B$ such that $A \neq B$.

Below we prove that equation (2.2) is not Darboux integrable if $A B(A-B) \neq 0$. To do this, we employ two-point invertible transformations. Alike transformations for differentialdifference and continuous analogues of the quad-graph equations were introduced in works [12] and [13], respectively. For discrete equations (1.2), such transformations were, in fact, used in [14] and then considered in a more explicit form in [15]. In particular, these transformations for 2.2 were briefly given in [15], and we reproduce them in the next two paragraphs with detailed calculations to make it easier for the reader to check them.

The mentioned transformation for $(2.2)$ is as follows. We can rewrite $(2.2)$ as

$$
u_{n+1, m+1} \frac{u_{n, m+1}+A}{u_{n, m+1}}=\frac{\left(u_{n+1, m}+B\right)\left(u_{n, m}+A\right)}{u_{n, m}+B} .
$$

We denote

$$
v_{n, m}=u_{n+1, m} \frac{u_{n, m}+A}{u_{n, m}}-A .
$$

Then equations (2.4) implies that

$$
v_{n, m+1}=\frac{\left(u_{n+1, m}+B\right)\left(u_{n, m}+A\right)}{u_{n, m}+B}-A
$$

by virtue (i.e., on solutions) of the equation (2.2). The next step is to resolve the system (2.5)(2.6) with respect to $u_{n, m}$ and $u_{n+1, m}$, that is, to express $u_{n, m}$ and $u_{n+1, m}$ in terms of $v_{n, m}$ and $v_{n, m+1}$. Resolving 2.5 with respect to $u_{n+1, m}$, we obtain

$$
u_{n+1, m}=\frac{u_{n, m}\left(v_{n, m}+A\right)}{u_{n, m}+A} .
$$

The substitution of 2.7 into 2.6 results in

$$
v_{n, m+1}=\frac{u_{n, m}\left(v_{n, m}+B\right)}{u_{n, m}+B} .
$$


Resolving 2.8 with respect to $u_{n, m}$, we get

$$
u_{n, m}=\frac{B v_{n, m+1}}{B+v_{n, m}-v_{n, m+1}} .
$$

Substituting (2.9) into (2.7), we find:

$$
u_{n+1, m}=\frac{B v_{n, m+1}\left(v_{n, m}+A\right)}{A\left(v_{n, m}+B\right)+(B-A) v_{n, m+1}} .
$$

If we shift 2.9$)$ by 1 in $n$ and compare the result with 2.10$)$, we obtain the equality

$$
\frac{B v_{n+1, m+1}}{B+v_{n+1, m}-v_{n+1, m+1}}=\frac{B v_{n, m+1}\left(v_{n, m}+A\right)}{A\left(v_{n, m}+B\right)+(B-A) v_{n, m+1}} .
$$

Resolving (2.11) with respect to $v_{n+1, m+1}$, we see that

$$
v_{n+1, m+1}\left(v_{n, m}+B\right)\left(v_{n, m+1}+A\right)=v_{n, m+1}\left(v_{n+1, m}+B\right)\left(v_{n, m}+A\right) .
$$

The last equation coincides with (2.2) up to the change of notation $u \rightarrow v$. Thus, transformation (2.5) maps solutions of (2.2) into solutions of (2.2) again, that is, 2.5) is a Bäcklund autotransformation for the Hietarinta equation 2.2 .

Repeating the arguing in the previous paragraph in the inverse order, it is easy to confirm that

$$
\bar{v}_{n, m}=\frac{B u_{n, m+1}}{B+u_{n, m}-u_{n, m+1}},
$$

is in fact 2.9 if we replace the notations as $u \rightarrow \bar{v}, v \rightarrow u$ and this is also an auto-transformation for equation (2.2). Indeed, starting from (2.11) and introducing $u_{n, m}$ by (2.9), we obtain (2.10). Equation (2.8) appears by solving (2.9) with respect to $v_{n, m+1}$, and (2.7) does by substituting (2.8) into (2.10). Equation (2.7) implies (2.5), and (2.6) is obtained by substituting (2.5) into (2.8). The comparison of (2.5) and (2.6) gives us the Heitarinta equation in form (2.4).

Remark 2.1. Under the condition $B \neq 0$, all formulae (2.4)-(2.11) remain valid even if $A=0$ or $A=B$. Thus, 2.5 and

$$
\bar{v}_{n, m}=\frac{B u_{n, m+1}}{B+u_{n, m}-u_{n, m+1}}
$$

are auto-transformations for (2.2) in these cases, too. But (2.2) loses the dependence of $u_{n, m+1}$ or $u_{n, m}$ if $A=0$ or $A=B$, respectively.

\section{TRANSFORMATION OF INTEGRALS}

In this section, we formulate some general propositions that are applicable, in particular, to Hietarinta equation (2.2). The key propositions, Lemmata 3.1, 3.2, were proved in [16] in the case of autonomous integrals. For the reader's convenience and to demonstrate that these propositions remain valid for non-autonomous integrals too, below we reproduce corresponding (slightly modified) proofs from [16].

Lemma 3.1. Let there exist functions $\varphi(x, y), \varphi_{y} \neq 0$, and $\psi(x, y)$ such that the right-hand side of (1.2) satisfies the relation

$$
\varphi\left(u_{n, m+1}, F\left(u_{n, m}, u_{n+1, m}, u_{n, m+1}\right)\right)=\psi\left(u_{n, m}, u_{n+1, m}\right),
$$

that is, $\varphi\left(u_{n, m+1}, u_{n+1, m+1}\right)=\psi\left(u_{n, m}, u_{n+1, m}\right)$ on solutions of 1.2 . In addition, let the identity $\varphi(x, y)=v$ be uniquely solvable for $y$. Then each $p$-th order $n$-integral for the equation (1.2) can be written in the form

$$
\Phi_{n, m}\left(\varphi\left(u_{n, m}, u_{n+1, m}\right), \varphi\left(u_{n+1, m}, u_{n+2, m}\right), \ldots, \varphi\left(u_{n+p-1, m}, u_{n+p, m}\right)\right) .
$$


Note that Lemma 3.1 only defines the form of the integrals (if they exist) but does not guarantee their existence.

Proof. Resolving the identity $\varphi\left(u_{n, m}, u_{n+1, m}\right)=v$ with respect to $u_{n+1, m}$, we obtain

$$
u_{n+1, m}=g\left(\varphi\left(u_{n, m}, u_{n+1, m}\right), u_{n, m}\right) .
$$

Let (1.2) admit a $p$-th order $n$-integral $\Omega_{n, m}$. Using expression (3.3) as well as its consequences derived by shifts in $n$, we rewrite $\Omega_{n, m}$ in terms of $u_{n, m}, \varphi\left(u_{n, m}, u_{n+1, m}\right)$ and its shifts in $n$ :

$$
\Omega_{n, m}=\Phi_{n, m}\left(u_{n, m}, \varphi\left(u_{n, m}, u_{n+1, m}\right), \varphi\left(u_{n+1, m}, u_{n+2, m}\right), \ldots, \varphi\left(u_{n+p-1, m}, u_{n+p, m}\right)\right) .
$$

The relation (3.1) implies that $\varphi\left(u_{n+j, m+1}, u_{n+j+1, m+1}\right)=\psi\left(u_{n+j, m}, u_{n+j+1, m}\right)$ on solutions of (1.2). Therefore, the shift of (3.4) in $m$ gives:

$$
\Omega_{n, m+1}=\Phi_{n, m+1}\left(u_{n, m+1}, \psi\left(u_{n, m}, u_{n+1, m}\right), \psi\left(u_{n+1, m}, u_{n+2, m}\right), \ldots, \psi\left(u_{n+p-1, m}, u_{n+p, m}\right)\right) .
$$

Comparing the last identity and (3.4), we see that $\Omega_{n, m}$ satisfies the defining relation (1.3) only if the function $\Phi_{n, m+1}$ in (3.4) is independent of its first argument for all $n$ and $m$.

Let us consider the equation (1.7) as an illustrative example. Subtracting 1 from both sides of (1.7) and then replacing them with their reciprocal values, we rewrite this equation in the form

$$
\frac{u_{n, m+1}}{u_{n+1, m+1}-u_{n, m+1}}=\frac{u_{n, m}+1}{u_{n+1, m}-u_{n, m}} .
$$

Thus, (1.7) satisfies all conditions of Lemma 3.1 with

$$
\varphi\left(u_{n, m}, u_{n+1, m}\right)=\frac{u_{n, m}}{u_{n+1, m}-u_{n, m}}, \quad \psi\left(u_{n, m}, u_{n+1, m}\right)=\frac{u_{n, m}+1}{u_{n+1, m}-u_{n, m}} .
$$

This choice of $\varphi$ and $\psi$ is not most obvious but convenient for the reasoning in the next paragraph. It is easy to check that

$$
\frac{u_{n+2, m}-u_{n+1, m}}{u_{n+1, m}-u_{n, m}}=\frac{v_{n, m}+1}{v_{n+1, m}},
$$

where

$$
v_{n, m}=\varphi\left(u_{n, m}, u_{n+1, m}\right)=\frac{u_{n, m}}{u_{n+1, m}-u_{n, m}} .
$$

Note that the left-hand side of $(3.5)$ is an $n$-integral of $(1.7)$ and the right-hand side is the representation in the form 3.2 for this integral.

Applying the scheme of the invertible two-point transformations from Section 2, we see that

$$
v_{n, m}=\frac{u_{n, m}}{u_{n+1, m}-u_{n, m}}
$$

maps solutions of (1.7) into solutions of the equation (2.3) and the right-hand side of (3.5) is an $n$-integral for (2.3). The latter is a particular case of a more general fact formulated in the following Lemma.

Lemma 3.2. Let equation (1.2) satisfy the assumptions of Lemma 3.1, the functions $\varphi(x, y)$, $\psi(x, y)$ be functionally independent and the transformation $v_{n, m}=\varphi\left(u_{n, m}, u_{n+1, m}\right)$ map solutions of (1.2) into solutions of an equation

$$
v_{n+1, m+1}=Q\left(v_{n, m}, v_{n+1, n}, v_{n, m+1}\right) .
$$

Then equation (1.2) admits an n-integral of order $p>1$ only if equation (3.6) admits an $n$-integral of order $p-1$. 
Proof. Let $\Omega_{n, m}$ be a $p$-th order $n$-integral of $(1.2)$. Lemma 3.1 implies that

$$
\Omega_{n, m}=\Phi_{n, m}\left(v_{n, m}, v_{n+1, m}, \ldots, v_{n+p-1, m}\right),
$$

where $v_{i, j}=\varphi\left(u_{i, j}, u_{i+1, j}\right)$. Let $T_{n}^{k}$ denote the combination of the shift by $k$ in $n$ and the elimination of the variables $v_{n+i, m+1}, i>0$, by using equation (3.6). Since $v_{n, m}$ satisfies (3.6) for each solution of $(1.2)$, we have

$$
\begin{aligned}
& v_{n+1, m+1}=T_{n}^{1}\left(v_{n, m+1}\right)=Q\left(v_{n, m}, v_{n+1, m}, v_{n, m+1}\right), \\
& v_{n+2, m+1}=T_{n}^{1}(Q)=Q\left(v_{n+1, m}, v_{n+2, m}, Q\left(v_{n, m}, v_{n+1, m}, v_{n, m+1}\right)\right), \\
& v_{n+k+1, m+1}=T_{n}^{k}(Q)=Q\left(v_{n+k, m}, v_{n+k+1, m}, T_{n}^{k-1}(Q)\right), \quad k>1 .
\end{aligned}
$$

Substituting these formulae into the defining relation for integral (3.7), we obtain

$$
\Phi_{n, m+1}\left(v_{n, m+1}, Q, \ldots, T_{n}^{p-2}(Q)\right)=\Phi_{n, m}\left(v_{n, m}, v_{n+1, m}, \ldots, v_{n+p-1, m}\right) .
$$

This identity holds as

$$
v_{n, m+1}=\varphi\left(u_{n, m+1}, u_{n+1, m+1}\right)=\psi\left(u_{n, m}, u_{n+1, m}\right),
$$

see $(3.1)$, and

$$
v_{n+\ell, m}=\varphi\left(u_{n+\ell, m}, u_{n+\ell+1, m}\right), \quad \ell=\overline{0, p-1},
$$

other variables $v_{i, j}$ are absent in (3.8). But $\psi\left(u_{n, m}, u_{n+1, m}\right)$ and $\varphi\left(u_{n+\ell, m}, u_{n+\ell+1, m}\right)$ are functionally independent and, hence, (3.8) should hold identically for arbitrary $v_{n, m+1}$ and $v_{n, m+\ell}$. Thus, $\Phi_{n, m}\left(v_{n, m}, v_{n+1, m}, \ldots, v_{n+p-1, m}\right)$ is an $n$-integral of the equation (3.6). Taking (3.7) into account, we see that $\Phi_{n, m}$ essentially depends on $v_{n, m}$ and $v_{n+p-1, m}$ if

$$
\frac{\partial \Omega_{n, m}}{\partial u_{n, m}} \neq 0 \quad \text { and } \quad \frac{\partial \Omega_{n, m}}{\partial u_{n+p, m}} \neq 0 .
$$

The integral $\Phi_{n, m}$ therefore has order $p-1$. The proof is complete.

Remark 3.1. In contrast to 1.2 , we do not assume

$$
\frac{\partial Q}{\partial v_{n+1, m}} \frac{\partial Q}{\partial v_{n, m+1}} \frac{\partial Q}{\partial v_{n, m}} \neq 0
$$

for (3.6). But this inequality follows from assumptions of Lemma 3.2. Indeed, substituting

$$
v_{n, m}=\varphi\left(u_{n, m}, u_{n+1, m}\right), \quad v_{n, m+1}=\psi\left(u_{n, m}, u_{n+1, m}\right)
$$

into (3.6), we obtain

$$
\psi\left(u_{n+1, m}, u_{n+2, m}\right)=Q\left(\varphi\left(u_{n, m}, u_{n+1, m}\right), \varphi\left(u_{n+1, m}, u_{n+2, m}\right), \psi\left(u_{n, m}, u_{n+1, m}\right)\right)
$$

and we see that the last relation holds only if $Q$ depends essentially on its second argument. The functional independence of $\varphi\left(u_{n+1, m}, u_{n+2, m}\right)$ and $\psi\left(u_{n+1, m}, u_{n+2, m}\right)$ excludes the case when $Q$ is independent of both first and third arguments. Differentiating relation (3.9) with respect to $u_{n, m}$, we see that this relation cannot hold if $Q$ is independent of its first or third arguments since $\varphi\left(u_{n, m}, u_{n+1, m}\right), \psi\left(u_{n, m}, u_{n+1, m}\right), \varphi\left(u_{n+1, m}, u_{n+2, m}\right)$ are functionally independent and these functions should essentially depend on their first argument to be compatible with both (3.1) and the inequality in (1.2).

The next proposition is a direct implication of Lemma 3.2 .

Theorem 3.1. Let equation (1.2) satisfy the assumptions of Lemma 3.1, the functions $\varphi(x, y), \psi(x, y)$ be functionally independent and the transformation $v_{n, m}=\varphi\left(u_{n, m}, u_{n+1, m}\right)$ map solutions of (1.2) into solutions of (1.2) again. Then equation $(1.2)$ does not admit $n$-integrals. 
Roughly speaking, the above theorem means that a quad-graph equation has no $n$-integrals if it admits a two-point invertible, in the sense of [13], [15], auto-transformation depending on the shift of $u_{n, m}$ in $n$.

Proof. We argue by contradiction and assume the contrary. Let 1.2 have an $n$-integrals of order $p$. If $p>1$, then, applying Lemma 3.2 several times, we obtain that 1.2 admits a first-order $n$-integral.

Each first-order integral of $(1.2)$ is of the form $\Phi_{n, m}\left(\varphi\left(u_{n, m}, u_{n+1, m}\right)\right)$ by Lemma 3.1, and (3.1) implies that the defining relation for this integral takes the form

$$
\Phi_{n, m+1}\left(\psi\left(u_{n, m}, u_{n+1, m}\right)\right)=\Phi_{n, m}\left(\varphi\left(u_{n, m}, u_{n+1, m}\right)\right) .
$$

But this contradicts the functional independence of $\psi$ and $\varphi$. The proof is complete.

Making the interchange $n \leftrightarrow m$ in Lemmata 3.1, 3.2, Theorem 3.1 and in their proofs, we also prove the following analogue of Theorem 3.1 for $m$-integrals.

Theorem 3.2. Let there exit functionally independent functions $\bar{\varphi}(x, y)$ and $\bar{\psi}(x, y)$ such that the right-hand side of $(1.2)$ satisfies the relation

$$
\bar{\varphi}\left(u_{n+1, m}, F\left(u_{n, m}, u_{n+1, m}, u_{n, m+1}\right)\right)=\bar{\psi}\left(u_{n, m}, u_{n, m+1}\right),
$$

that is, $\bar{\varphi}\left(u_{n+1, m}, u_{n+1, m+1}\right)=\bar{\psi}\left(u_{n, m}, u_{n, m+1}\right)$ on solutions of $(1.2)$. In addition, let the equation $\bar{\varphi}(x, y)=\bar{v}$ be uniquely solvable for $y$. Then equation (1.2) does not admit $m$-integrals if the transformation $\bar{v}_{n, m}=\bar{\varphi}\left(u_{n, m}, u_{n, m+1}\right)$ maps solutions of (1.2) into solutions of (1.2).

As it is demonstrated in Section 2, equation (2.2) satisfies all assumptions of Theorems 3.1. 3.2 in the case $A B(A-B) \neq 0$. The corresponding functions $\varphi, \psi, \bar{\varphi}$ and $\bar{\psi}$ are

$$
\begin{array}{ll}
\varphi\left(u_{n, m}, u_{n+1, m}\right)=u_{n+1, m} \frac{u_{n, m}+A}{u_{n, m}}-A, & \psi\left(u_{n, m}, u_{n+1, m}\right)=\frac{\left(u_{n+1, m}+B\right)\left(u_{n, m}+A\right)}{u_{n, m}+B}-A, \\
\bar{\varphi}\left(u_{n, m}, u_{n, m+1}\right)=\frac{B u_{n, m+1}}{B+u_{n, m}-u_{n, m+1}}, & \bar{\psi}\left(u_{n, m}, u_{n, m+1}\right)=\frac{B u_{n, m+1}\left(u_{n, m}+A\right)}{A\left(u_{n, m}+B\right)+(B-A) u_{n, m+1}} .
\end{array}
$$

Thus, taking Proposition 2.1 into account, we make the following conclusion.

Proposition 3.1. Hietarinta equation (1.1) has no $n$-and m-integrals if $(a-c)(b-d) \neq 0$.

Theorems 3.1 and 3.2 are also applicable to the linear equation

$$
u_{n+1, m+1}=\alpha u_{n+1, m}+\beta u_{n, m+1}+\gamma u_{n, m}
$$

with constant coefficients $\alpha, \beta$ and $\gamma$. Indeed,

$$
\begin{array}{ll}
\varphi\left(u_{n, m}, u_{n+1, m}\right)=u_{n+1, m}-\beta u_{n, m}, & \psi\left(u_{n, m}, u_{n+1, m}\right)=\alpha u_{n+1, m}+\gamma u_{n, m}, \\
\bar{\varphi}\left(u_{n, m}, u_{n, m+1}\right)=u_{n, m+1}-\alpha u_{n, m}, & \bar{\psi}\left(u_{n, m}, u_{n, m+1}\right)=\beta u_{n, m+1}+\gamma u_{n, m}
\end{array}
$$

for this equation. The transformations $v_{n, m}=u_{n+1, m}-\beta u_{n, m}, \bar{v}_{n, m}=u_{n, m+1}-\alpha u_{n, m}$, which coincide with the discrete Laplace transformations (see [17], [9]), map solutions of (3.10) into solutions of $(3.10)$ again. Thus, Theorems 3.1 and 3.2 imply that 3.10$)$ has no integrals in the case $\gamma+\alpha \beta \neq 0$ because both the pairs $\varphi, \psi$ and $\bar{\varphi}, \psi$ are functionally independent under this condition.

Remark 3.2. As it was demonstrated in [9], [10], to vanish a Laplace invariant is a necessary condition for the existence of autonomous integrals of (1.2). Analyzing the proof of Proposition 2 in [10], we can make sure that this necessary condition remains valid for nonautonomous integrals of autonomous equations. Since all Laplace invariants of (3.10) are equal to $\gamma+\alpha \beta$, we see that the Laplace invariants provides an alternative way to prove the absence 
of integrals for equation (3.10) in the case $\gamma+\alpha \beta \neq 0$. And this provides another way to prove the Darboux non-integrability of the generic Hietarinta equation if we employ the fact [11] that the transformation $u_{n, m}=\frac{v_{n+1, m}}{v_{n, m}}-A$ maps solutions of the linear equation

$$
v_{n+1, m+1}=v_{n+1, m}+A v_{n, m+1}+(B-A) v_{n, m}
$$

into solutions of (2.2). Indeed, since all Laplace invariants of (3.11) are equal to $B$, this linear equation is not Darboux integrable in the case $B \neq 0$ and the Darboux integrability of the corresponding Hietarinta equation (2.2) contradicts the following obvious statement, cf. Lemma 1 in [7].

Proposition 3.2. Let a transformation

$$
u_{n, m}=\phi\left(v_{n, m}, v_{n+1, m}, \ldots, v_{n+k, m}\right), \quad k>0, \quad \frac{\partial \phi}{\partial v_{n, m}} \frac{\partial \phi}{\partial v_{n+k, m}} \neq 0,
$$

map solutions of an equation

$$
v_{n+1, m+1}=Q\left(v_{n, m}, v_{n+1, m}, u_{n, m+1}\right)
$$

into solutions of (1.2) and let the equation (1.2) admit an $n$-integral of order $p$. Then the equation (3.13) possesses an $n$-integral of order $p+k$.

Proof. Let $\Omega_{n, m}$ be an $n$-integral for (1.2). Then defining relation (1.3) holds for each solution of 1.2 and, in particular, for all solutions obtained by formula 3.12 from solutions of (3.13). Therefore,

$$
\Omega_{n, m}\left(\phi\left(v_{n, m}, v_{n+1, m}, \ldots, v_{n+k, m}\right), \ldots, \phi\left(v_{n+p, m}, v_{n+p+1, m}, \ldots, v_{n+p+k, m}\right)\right)
$$

is an $n$-integral of $(3.13)$. The proof is complete.

Thus, the Hietarinta equation gives an example of a quad-graph equation that is linearizable but is not Darboux integrable.

It should be noted that Remark 3.2 describes a sketch for the proof of the Darboux nonintegrability of 2.2 in a way very similar to that was used in [7] for estimating the minimal orders of integrals for a particular linearizable quad-graph equation. In the present paper, the author prefers another way that does not require introducing the Laplace invariants and therefore seems to be more self-contained.

\section{REFERENCES}

1. V.E. Adler, A.I. Bobenko, Yu.B. Suris. Discrete nonlinear hyperbolic equations. Classification of integrable cases // Funkt. Analiz Prilozh. 43:1, 3-21 (2009). [Funct. Anal. Appl. 43, 3-17 (2009).]

2. J. Hietarinta. A new two-dimensional lattice model that is 'consistent around a cube'// J. Phys. A: Math. Gen. 37:6, L67-L73 (2004).

3. G. Gubbiotti, C. Scimiterna. Reconstructing a lattice equation: a non-autonomous approach to the Hietarinta equation // SIGMA 14, 004 (2018).

4. S.Ya. Startsev. Darboux integrable discrete equations possessing an autonomous first-order integral // J. Phys. A: Math. Theor. 47:10, 105204, 16pp (2014).

5. R.N. Garifullin, R.I. Yamilov. Generalized symmetry classification of discrete equations of a class depending on twelve parameters // J. Phys. A: Math. Theor. 45:34, 345205 (2012).

6. A.V. Zhiber, V.V. Sokolov. Exactly integrable hyperbolic equations of Liouville type // Uspekhi Matem. Nauk. 56:1, 63-106 (2001). [Russ. Math. Surv. 56:1, 61-101 (2001).]

7. R.N. Garifullin, R.I. Yamilov. Examples of Darboux integrable discrete equations possessing first integrals of an arbitrarily high minimal order // Ufimskij Matem. Zhurn. 4:3, 177-183 (2012). [Ufa Math. J. 4:3, 174-180 (2012).] 
8. I.T. Habibullin. Characteristic algebras of fully discrete hyperbolic type equations // SIGMA 1, $023(2005)$.

9. V.E. Adler, S.Ya. Startsev. Discrete analogues of the Liouville equation // Teor. Mat. Fiz. 121:2, 271-285 (1999). [Theor. Math. Phys. 121:2, 1484-1495 (1999).]

10. S.Ya. Startsev. Relationships between symmetries depending on arbitrary functions and integrals of discrete equations // J. Phys. A: Math. Theor. 50:50, 50LT01 (2017).

11. A. Ramani, N. Joshi, B. Grammaticos, T. Tamizhmani. Deconstructing an integrable lattice equation // J. Phys. A: Math. Gen. 39:8, L145-L149 (2006).

12. R.I. Yamilov. Invertible changes of variables generated by Bäcklund transformations // Teor. Mat. Fiz. 85:3, 368-375 (1990). [Theor. Math. Phys. 85:3, 1269-1275 (1990).]

13. V.V. Sokolov, S.I. Svinolupov. On nonclassical invertible transformation of hyperbolic equations // Eur. J. Appl. Math. 6:2, 145-156 (1995).

14. R.I. Yamilov. Construction scheme for discrete Miura transformation // J. Phys. A: Math. Gen. 27:20, 6839-6851 (1994).

15. S.Ya. Startsev. On non-point invertible transformations of difference and differential-difference equations // SIGMA 6, 092 (2010).

16. S.Ya. Startsev. Non-Point Invertible Transformations and Integrability of Partial Difference Equations // SIGMA 10, 066 (2014).

17. S.P. Novikov, I.A. Dynnikov. Discrete spectral symmetries of low-dimensional differential operators and difference operators on regular lattices and two-dimensional manifolds // Uspekhi Matem. Nauk. 52:5, 175-234 (1997). [Russ. Math. Surv. 52:5, 1057-1116 (1997).]

Sergey Yakovlevich Startsev,

Institute of Mathematics,

Ufa Federal Research Centre, RAS,

Chernyshevsky str., 112,

450008, Ufa, Russia

E-mail: startsev@anrb.ru 